\title{
Structure and dynamics in nanoionic materials
}

\author{
Alan V. Chadwick*, Shelley L.P. Savin \\ Functional Materials Group, School of Physical Sciences, University of Kent, Canterbury, Kent, CT2 7NR, UK
}

Received 5 May 2006; received in revised form 26 June 2006; accepted 31 July 2006

\begin{abstract}
Nanomaterials, materials with particle dimensions less than $100 \mathrm{~nm}$, show a range of unusual properties when compared with their bulk counterparts. Atomic transport is one of these properties and nanomaterials have been reported as having exceptionally high diffusion coefficients. In the case of ionic materials the atomic transport is important in a number of technological applications where they are used as solid electrolytes, for example in sensors, batteries and fuel cells. Hence ionic nanomaterials often referred to as nanoionics, can offer the means of producing electrolytes with improved performance. This contribution will examine the mechanisms of atomic transport in nanoionics in two model materials, zirconia and lithium niobate. Since an understanding of these mechanisms is dependent on knowledge of the microstructure of the materials consideration will also be given to the structural characterisation of the materials, with a focus on X-ray absorption spectroscopy. The use of this technique to characterise mesoporous a- $\mathrm{Fe}_{2} \mathrm{O}_{3}$ is also discussed.
\end{abstract}

(C) 2006 Elsevier B.V. All rights reserved.

Keywords: Nanoionics; Diffusion; XAS; Nanocrystals; Oxides

\section{Introduction}

Nanomaterials are systems that contain particles with one dimension in the nanometre regime. Currently there is intense interest from biologists, chemists, physicists and engineers in the application of these materials, so-called nanotechnology, which is sometimes referred to as 'the next industrial revolution' [1]. The reason for the interest is the unusual properties, very often with useful applications, that are exhibited by these materials when compared to their bulk counterparts [2-10]. In this article we will focus on rather simple ionic solids with dimensions predominantly less than $100 \mathrm{~nm}$, materials now known as nanoionics. In these systems the origin of the unusual properties is twofold; (i) the fact that the dimensions of the particles approaches, or becomes smaller than, the critical length for certain phenomena (e.g. the de Broglie wavelength for the electron, the mean free path of excitons, the distance required to form a Frank-Reed dislocation loop, thickness of

\footnotetext{
* Corresponding author.

E-mail address: a.v.chadwick@kent.ac.uk (A.V. Chadwick).
}

the space-charge layer, etc.) and (ii) surface effects dominate the thermodynamics and energetics of the particles (e.g. crystal structure, surface morphology, reactivity, etc.). The second factor can lead to nanocrystals adopting different morphologies to bulk crystals with different exposed lattice planes leading to an extraordinary surface chemistry $[11,12]$ and catalytic activity $[13,14]$. The importance of surfaces and boundaries in nanocrystalline systems is demonstrated in Fig. 1, which shows the fraction of atoms in these regions as a function of grain size.

Unusually rapid diffusion of atoms in nanocrystalline metals was recognised in early studies $[9,15,16]$. In turn this will affect a wide range of physical and chemical properties of which the enhanced plasticity of metal and ceramic compacts has attracted considerable attention [17]. Fast diffusion in ionic and semiionic solids has important technological implications for greatly improved materials in a range of applications such as electrolytes and electrodes for batteries and fuel cells, and chemical sensors [18-23]. As a result there has been considerable effort to quantify the extent of the diffusion enhancement and to determine the nature of the fundamental processes involved. However, until relatively recently, surveys of the experimental data of the diffusion in nanoionic materials 


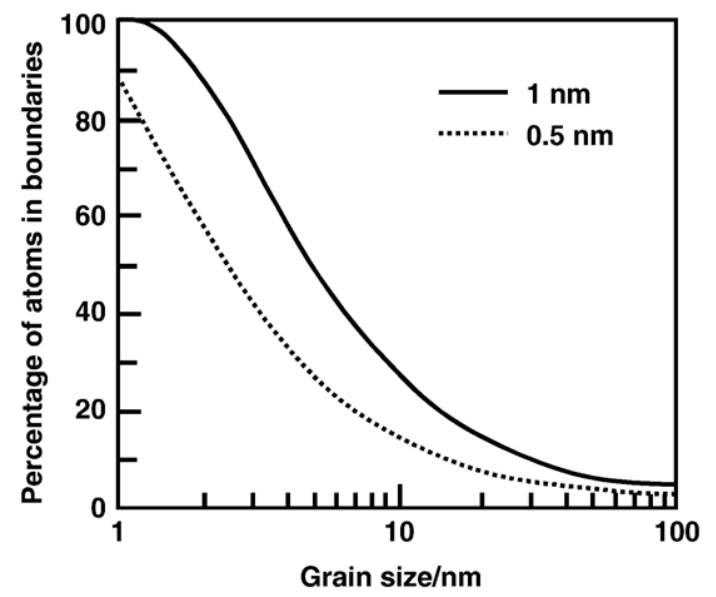

Fig. 1. Percentage of atoms in grain boundaries as a function of grain size assuming boundary widths of 0.5 and $1 \mathrm{~nm}$.

showed that the results were not consistent, with variations even for the same system [24]. A breakthrough came with the work of Sata and co-workers who provided unambiguous experimental evidence of enhanced diffusion in layered nanocrystalline films of $\mathrm{BaF}_{2}$ and $\mathrm{CaF}_{2}$ [25]. Nevertheless, as recent articles have stressed [26,27], the volume of reliable experimental information is still sparse and the detailed understanding of the phenomenon is poor. A major problem is in the preparation of reliable samples and that the properties are very dependent on the synthetic route.

This contribution will consider the most recent experimental studies on ionic transport in nanoionics. Given the importance of the preparation route particular attention will be paid to the characterisation of samples and the relationship between microstructure and the preparative route. Particular attention will be paid to the application of X-ray absorption spectroscopy, $\mathrm{XAS}$, as a probe of microstructure and some new results will be presented for some model systems.

\section{Methodology}

\subsection{Sample preparation}

As will be shown later the preparation route of nanocrystalline samples determines the microstructure and hence the atomic transport behaviour so a brief overview of the methods will be given here. A very wide variety of methods have been employed to produce nanocrystalline samples and only the more commonly used ones will be mentioned. Inert gas condensation (IGC) has been extensively used to fabricate metallic and metal oxide powders with a well-defined and narrow size distribution $[2,28]$. The amount of material that can be produced is relatively small and there have been several modifications to increase the yield using sputtering methods [29-32]. Spray pyrolysis is a fairly general method of producing nanocrystalline oxides. In this case a solution of a chemical precursor is dispersed into the gas phase as aerosol droplets. The droplets are then transported to a hot zone where they are decomposed to form oxide particles. This method has relatively wide applicability and has been used to prepare several metal oxide nanoparticles such as $\mathrm{ZnO}, \mathrm{ZrO}_{2}$ and $\mathrm{Al}_{2} \mathrm{O}_{3}$ [33].

Sol-gel procedures have been used for many years to produce oxides and ceramics offering control over the structure and composition at the molecular level $[34,35]$. The usual procedure is to subject a metal alkoxide, $\mathrm{M}(\mathrm{OR})_{x}$ to controlled hydrolysis, leading to replacement of the OR group by $\mathrm{OH}$. This leads to the formation of a sol, very small colloidal particles, which then condense to form a gel, an inter-connected network. The gel is then dried and the final product can be either oxide (as in the case of silicon tetraethyl orthosilicate) or hydroxide (zirconium iso-propoxide) or a mixed methoxy-hydroxide (as in the case of magnesium methoxide). Thus the final step in the formation of the oxide is calcination at high temperature. This step is difficult to control and presents two major problems, as exemplified by recent work on $\mathrm{ZrO}_{2}$ [36]. If the calcining temperature is too low then all of the residual $\mathrm{OH}$ groups may not be completely removed from the material. If the calcination temperature is too high then the particles will grow and the nanocrystallites will be lost. The surface energy of nanocrystals is such that relatively moderate temperatures $\left(\sim 400{ }^{\circ} \mathrm{C}\right.$ in the case of most oxides) will lead to measurable grain growth over the period of a few minutes [37].

An apparently completely general method of producing all forms of nanocrystals is by mechanical attrition. This involves taking bulk material and reducing the grain size in a high-energy ball mill [38-41]. The advantages of ball milling are the fact that almost every material is accessible, that large amounts can be produced and that the average grain size can easily be varied by choice of the milling time. In addition, it is possible to produce materials in situ in the ball mill by double decomposition reactions $[42,43]$. This method is therefore useful when many different materials are to be compared. One disadvantage of ball milling is that abrasion of the milling media may occur. This has to be minimized by choosing appropriate materials for the milling vial and balls, respectively. A further disadvantage is that the milling can produce amorphous debris, to the extent that recent work on ball milled $\mathrm{Al}_{2} \mathrm{O}_{3}$ indicated that the sample consisted of nanocrystalline grains embedded in amorphous material [44].

\subsection{Microstructure invstigation}

The microstructure is the key to the properties of nanocrystalline materials. It was seen earlier that simple geometric considerations lead to the conclusion that a large fraction of the atoms in a nanocrystal are in the surface (see Fig. 1). However, the crucial questions are what is the nature of the surface, in terms of the level of atomic order, and what is the structure of the interface between grains. In both cases, two extreme possibilities have been considered. One extreme is that there is extensive disorder in an interface that is several atoms in width. In some of the early work on nanocrystals this was intuitively assumed to be the case and the interfaces were referred to as 'gas-like' or 'liquid-like'. This structure would clearly account for rapid diffusion in nanocrystalline samples. The alternative view is that the interface is similar to a grain 
boundary in normal bulk materials. In this case the interfaces would exhibit usual behaviour, although they would be present in unusually large number.

High resolution TEM can provide the microstructural details. TEM measurements on nanocrystalline ceria showed that the grains had a high degree of perfection and were separated by sharp, boundaries [45]. Unfortunately the data from HRTEM studies are still relatively sparse and other structural techniques have had to been used to explore the microstructure, such as electron diffraction [46], positron annihilation spectroscopy [47] and extended X-ray absorption fine structure (EXAFS) measurements [48-50].

EXAFS are the oscillations in the X-ray absorption (a plot of absorption coefficient, $\mu$, versus incident photon energy) that occur beyond the absorption edge for the emission of a core ( $\mathrm{K}$ or L shell) electron [51-53]. The oscillations arise from the emitted photoelectron wave being backscattered and interfering with the outgoing wave. If the two waves are in phase there will be constructive interference, a lower final state energy and a higher probability for absorption. If the two waves are out of phase then there will be destructive interference, higher final state energy and a lower probability for absorption. Thus as the incident photon energy increases so does the energy of the emitted photoelectron with consequential changes on its wavelength. Since the distance between the target atom and its neighbours is fixed there will be shifts in and out of phase and hence the observation of the EXAFS oscillations. The intensity of the oscillations depends on the number and type of neighbours giving rise to the backscattering and an EXAFS Debye-Waller factor (an uncertainty in the distance between target and scattering atoms). EXAFS does not rely on long-range order and is sensitive to the local environment of the target atom out to $5 \AA$. The Fourier transform of the EXAFS yields a partial radial distribution function in real space with peak areas proportional to average coordination numbers and the Debye-Waller factors.

For a nanocrystalline sample the EXAFS signal could be attenuated for two reasons: (i) the particle is so small that the average coordination numbers for the neighbouring shells is reduced or (ii) there is sufficient disorder in the sample (e.g. at the interfaces) that the Debye-Waller factors are increased. At first sight it would appear that EXAFS has little to offer as a microstructural probe, however for (i) to be operative the particle size has to be very small, typically less than $5 \mathrm{~nm}$. Thus, in principle, EXAFS can probe disorder in the interfaces of nanocrystals. However, the results have been very confusing and the subject of much argument. The EXAFS data for $\mathrm{ZrO}_{2}$ represent a typical example. There have been several EXAFS studies of this system, which claim evidence for disordered interfaces in nanocrystalline samples, i.e. an attenuation of the EXAFS for the $\mathrm{Zr}-\mathrm{Zr}$ correlation [54,55]. However, similar measurements on carefully prepared films, with particle sizes down to $6 \mathrm{~nm}$ found the EXAFS was indistinguishable from the bulk [56] and great care was taken to ensure all hydroxyl species were removed from the sample. Other studies of nanocrystalline materials have showed the preparation methods strongly affect the EXAFS data [36,51,56-58]. We will present below some new results of EXAFS studies of nanoionics.

\subsection{Ion transport invstigation}

A very wide range of techniques can be used to probe atomic transport. Elegant surveys of the methods can be found in earlier work [26,59-61]. A useful division is into macroscopic techniques, which measure the effect of long-range motion of atoms, and microscopic techniques, which measure jump frequencies of atoms. Tracer diffusion is the classical macroscopic technique [61]. In these experiments an isotopic tracer of the atom under study is diffused into the sample for a known time at a fixed temperature. Sections are then removed from the sample, the sections analysed for the tracer concentration, the penetration profile determined and $D$ determined from the boundary conditions [60]. For penetration depths larger than $1 \mu \mathrm{m}$ classical radiotracer techniques can be used, which implies mechanical sectioning of the specimen and subsequent measurement of radioactivity of the sections. By contrast, SIMS (secondary ion mass spectrometry) profiling is applicable for penetration depths smaller than $1 \mu \mathrm{m}$ [62]. The surface of the specimen is bombarded with a beam of primary ions, which results in a continuous atomisation of the sample. The sputtered secondary ions can then be detected in a mass spectrometer. An advantage of the tracer technique is that since the profile is determined it is often possible to separate out different diffusion process (e.g. bulk, grain boundary, surface diffusion, etc.) provided they have sufficiently different diffusivities.

Nuclear magnetic resonance (NMR) spectroscopy offers a wide range of methods for studying diffusion in the solid state $[63,64]$. If the diffusion is sufficiently fast in the solid $\left(D>10^{-13} \mathrm{~m}^{2} \mathrm{~s}^{-1}\right)$ then field gradient NMR methods can be employed. In this case, the nuclear spin is essentially used as a label (like a tracer), to follow the motion of the atoms over many jump distances. The diffusion coefficient can be determined directly from the measurement without the need to resort to a theoretical model. Thus this is a macroscopic method. The diffusive motions of the nuclei can affect the relaxation times of the nuclear spins, following a perturbance of the spin system by the application of a magnetic field. In simple terms, the moving spins will create oscillating magnetic fields that will interact with the spin system. Thus the NMR relaxation times $T_{1}$ (spin-lattice relaxation time), $T_{2}$ (spin-spin relaxation time), $T_{1 \rho}$ (spinlattice relaxation time in the rotating frame), etc., and line widths $\left(\propto 1 / T_{2}\right)$ can all provide information on diffusion. However, the time scale of the measurement is very short, such that the atoms traverse very few atomic distances and NMR relaxation time is a microscopic method. Except in special cases it is very difficult to obtain accurate values of $D$ from the measured relaxation times due to complexities in the theoretical models [64]. However, relative values are precise and accurate values of $Q$ can be evaluated. Before leaving the NMR techniques it is worth noting that for a number of particularly important elements they provide a convenient (in some cases the only) method of studying atomic diffusion. These include ${ }^{7} \mathrm{Li},{ }^{17} \mathrm{O}$ and ${ }^{19} \mathrm{~F}$, elements where the radiotracers are non-existent or very short-lived.

For ionic solids the measurement of the ionic conductivity, $\sigma$, has long provided a method of studying the atomic diffusion [65-67]. The early studies were restricted to measurements on 
single crystals, however this was very restricting in terms of the materials that could be investigated. Impedance spectroscopy is the measurement of the complex impedance over a wide range of A.C. frequency and is an important tool to study diffusion in solids [68-71]. The advantage of this technique is that it can be used to study polycrystalline and compacted samples and it can deconvolute the contributions from the different structural components of the sample like bulk material or grain boundaries.

\section{Results and discussion}

\subsection{Microstructure of nanoionics}

We have recently undertaken XAS experiments on three model nanocrystalline systems, zirconia, lithium niobate and iron oxide that exemplify the application of the technique in probing microstructure. The spectra were collected on stations at the CCLRC Daresbury Synchrotron Radiation Source (SRS) using procedures described in earlier work $[23,36,37,50,58,59,72,73]$.

\subsubsection{Zirconia}

Zirconium oxide, $\mathrm{ZrO}_{2}$, is one of the most studied materials due to its applications as an engineering ceramic and as an oxygen ion conductor for solid oxide fuel cells. It has also been the subject of numerous investigations as a nanoionic material due to the apparent ease of preparation. We use the term 'apparent' as the most common procedure of heating the hydroxide at modest temperature $\left(500{ }^{\circ} \mathrm{C}\right)$ for a short period (60 min) has been reported as a means of producing $10 \mathrm{~nm}$ particle sizes of the oxide [72]. Later work [36] has shown this procedure does not produce the pure oxide but material that contains a considerable fraction of hydroxyl species, sufficient to invalidate any EXAFS studies. A polymer precursor route can produce high quality thin films of nanocrystalline $\mathrm{ZrO} 2$. EXAFS studies $[50,56]$ of these samples have shown that with particle sizes as small as $6 \mathrm{~nm}$ there is no attenuation of the EXAFS signal and the interfaces between crystallites are similar to grain boundaries in bulk materials.

We have recently made new EXAFS measurements on powdered samples of pure nanocrystalline zirconia prepared by sol-gel procedures. We overcame the problem of grain growth during preparation by using silica, $15 \%$ by weight, to pin the grains [74]. The preparation involved the addition of TEOS to the zirconium alkoxide prior to the hydrolysis stage. The $\mathrm{ZrO}_{2}$ sample was in the tetragonal phase with a grain size of $10 \mathrm{~nm}$, even after calcining at $1000{ }^{\circ} \mathrm{C}$.

EXAFS spectra for the nanocrystalline $\mathrm{ZrO}_{2}$ were collected at a series of temperature down to $17 \mathrm{~K}$. The measurements were undertaken at low temperatures to remove any ambiguity about the possibility of the contributions from dynamic and static disorder to the attenuation of the EXAFS signal. Parallel data were also collected for a bulk sample of tetragonal zirconia that was prepared by the polymer precursor method with the addition of $8 \mathrm{~mol} \%$ yttria and calcined at $1000{ }^{\circ} \mathrm{C}$. The data for the two samples were virtually identical at all temperatures and only those at $17 \mathrm{~K}$ are shown in Fig. 2. This conclusively proves that the levels of both dynamic and static disorder in sol-gel prepared nanocrystalline $\mathrm{ZrO}_{2}$ are virtually the same as in the bulk material. Hence the interfaces between the grains are like grain boundaries in normal bulk samples.

Parallel studies were also made of ball-milled $\mathrm{ZrO}_{2}$ with a particle size of $13 \mathrm{~nm}$. X-ray diffraction showed that the sample was monoclinic [58]. For comparison data were also collected for a bulk, monoclinic sample (particle size $110 \mathrm{~nm}$ ). The Fourier transforms of the spectra collected at $17 \mathrm{~K}$ are shown in Fig. 3. There is a strong attenuation of the EXAFS signal for the ball-milled sample, represented by a dramatic reduction in the magnitude of the peak at $\sim 3.5 \AA$, the $\mathrm{Zr}-\mathrm{Zr}$ correlations. At this measurement temperature the differences can only be due to differences in the level of static disorder in the samples. We interpret this as the formation of significant levels of amorphous material in the ball-milled samples, a feature of this method of sample preparation $[44,73,75]$.

\subsubsection{Lithium niobate}

The report of unusually high lithium ion diffusion in ballmilled samples of $\mathrm{LiNbO}_{3}[76]$ was intriguing, as this is not
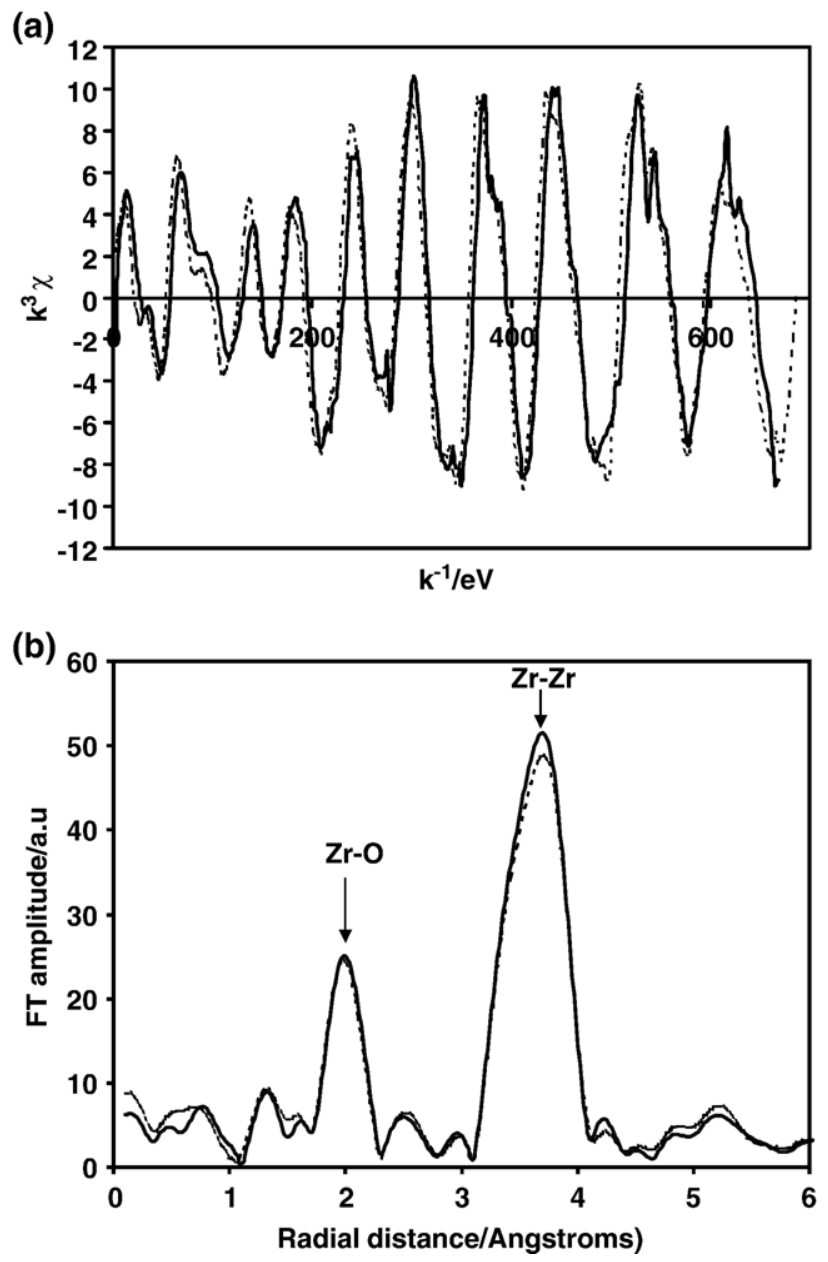

Fig. 2. The $\mathrm{Zr}$ K-edge EXAFS of tetragonal $\mathrm{ZrO}_{2}$ at $17 \mathrm{~K}$ : (a) the normalised EXAFS, (b) the Fourier transform which has been corrected with the phase shift of the first shell. The solid line $(-)$ is bulk material $(214 \mathrm{~nm})$, the dotted line $(\cdots \cdots \cdots)$ is sol-gel prepared nanocrystals $(10 \mathrm{~nm})$. 


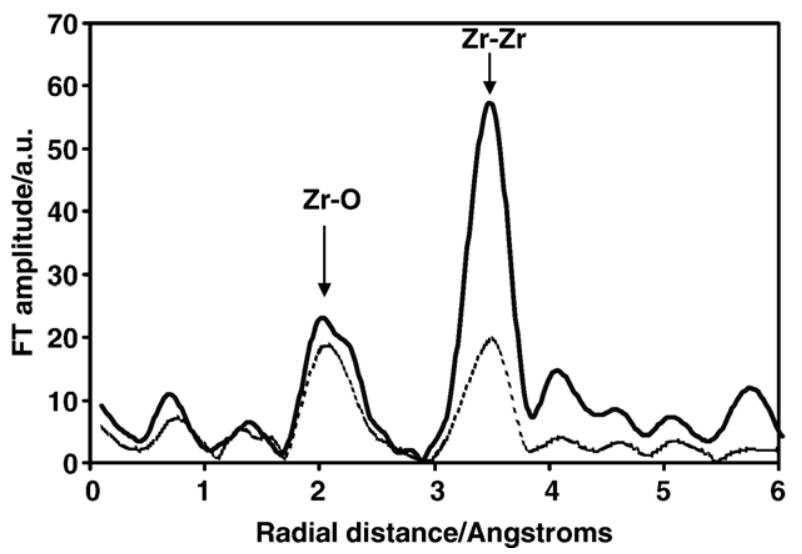

Fig. 3. The Fourier transform of $\mathrm{Zr} \mathrm{K}$-edge EXAFS of bulk and ball-milled monoclinic $\mathrm{ZrO}_{2}$ at $17 \mathrm{~K}$. The Fourier transform has been corrected with the phase shift of the first shell. The solid line $(--)$ is the bulk sample $(110 \mathrm{~nm})$, the dotted line (…...) is the ball-milled sample $(13 \mathrm{~nm})$.

normally regarded as a particularly good ionic conductor. We therefore undertook $\mathrm{Nb} \mathrm{K}$-edge XAS studies of nanocrystalline $\mathrm{LiNbO}_{3}$ prepared by sol gel methods from alkoxides and ballmilled $\mathrm{LiNbO}_{3}$ [77]. The results are shown in the form of the Fourier transforms in Fig. 4. The sol-gel sample (particle size $21 \mathrm{~nm}$ ) shows a transform which is very similar to bulk material, as would be expected as the particles relatively large and the average $\mathrm{CN}$ will not be reduced significantly. In contrast the ball-milled sample (particle size $23 \mathrm{~nm}$ ) exhibits considerably reduced peaks, even for the first peak, the $\mathrm{Nb}-\mathrm{O}$ correlation. We have interpreted this as due to the formation of a large fraction of amorphous material in the ball-milled sample, which we roughly estimate to be the order of $50 \%$. It is worth noting that these EXAFS findings were confirmed in a very recent study of lithium niobate by Heitjans and co-workers [78]. In addition

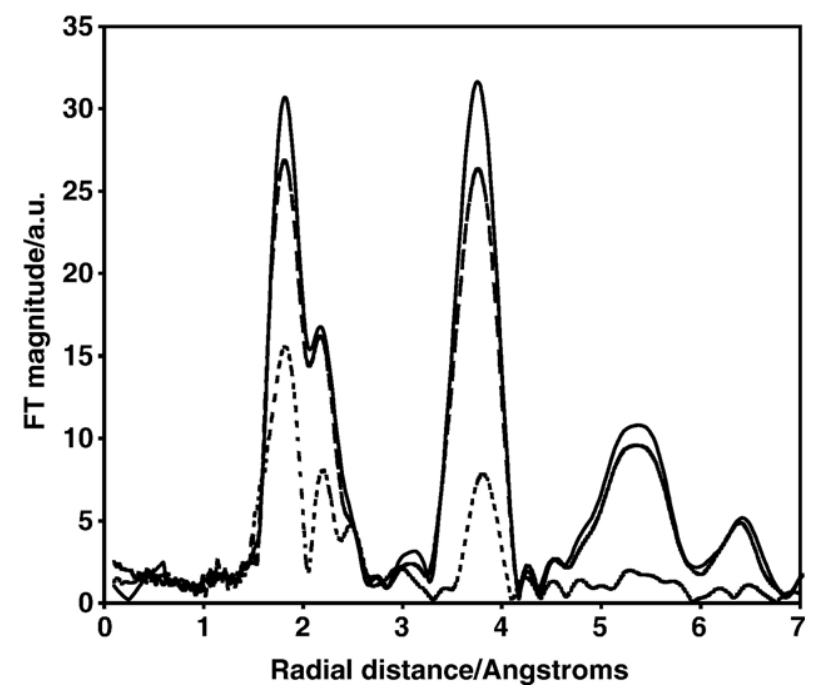

Fig. 4. The Fourier transform of the $\mathrm{Nb}$ K-edge EXAFS of $\mathrm{LiNbO}_{3}$. The transform has been corrected with the phase shift of the first shell. The solid line $(--)$ is a bulk sample, the long dashed line (----) is a sol-gel sample $(21 \mathrm{~nm})$, the short dashed line $(-)$ is a ball-milled sample $(50 \mathrm{~nm})$. they clearly showed the presence of amorphous material in the ball-milled sample by TEM measurements.

\subsubsection{Iron oxide, $\alpha-\mathrm{Fe}_{2} \mathrm{O}_{3}$}

Iron oxide, $\alpha-\mathrm{Fe}_{2} \mathrm{O}_{3}$, can be prepared as a mesoporous material with ordered or disordered pore walls, depending on the synthetic route, and has a variety of potential applications due to its magnetic properties [79]. We have performed extensive characterisation studies of these materials with wall thicknesses of $\sim 7 \mathrm{~nm}$ using a variety of methods of which the XAS has been particularly informative. The Fourier transforms of the Fe K-edge spectra of the samples are shown in Fig. 5. The similarity between the plots for the sample with ordered walls and the bulk sample, with only a slight reduction in the magnitude of the peaks for the $\mathrm{Fe}-\mathrm{Fe}$ correlations, indicates high degree crystallinity in the pore walls with crystallite sizes comparable to the wall thickness. In contrast the sample with disordered walls shows a dramatic reduction of the peaks due to the Fe-Fe correlations. The TEM and diffraction measurements for these samples show evidence of some crystallinity within the walls whilst the EXAFS suggests that the crystallites are very small (much smaller than the wall thickness) or the interfaces are highly disordered.

\subsection{Atomic transport in nanoionics}

The lack of reliable, quantitative data for atom transport in nanocrystals was highlighted at the outset of this contribution. There have been problems with the samples that have invalidated many of the conventional techniques. For example, sample porosity [80] has meant that the standard tracer sectioning method [60], the most reliable technique used in the study of diffusion in single crystals cannot be employed. In the case of thin film samples there can be problems of contamination from moisture [24]. However, there are now examples from the recent literature where the problems have been resolved and reliable information on transport has been achieved. We will describe two systems, namely zirconia, and lithium niobate, where there is good evidence of enhancement of the transport and an insight into the phenomenon.

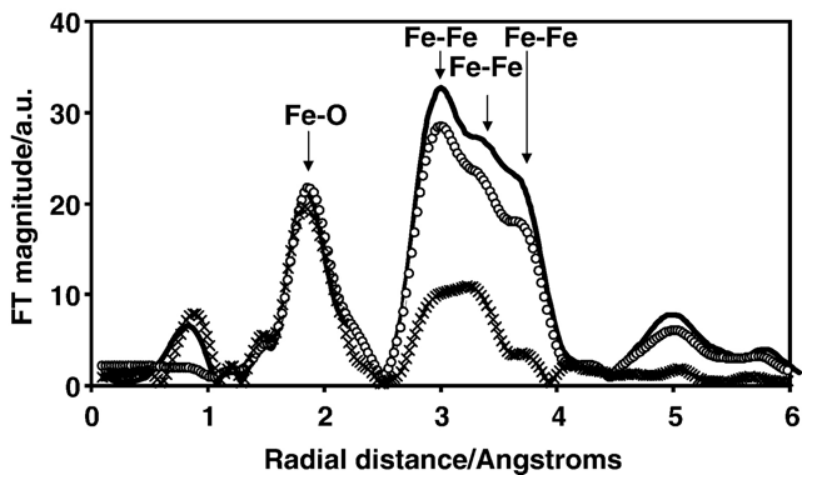

Fig. 5. The Fourier transform of the Fe K-edge EXAFS of $\alpha-\mathrm{Fe}_{2} \mathrm{O}_{3}$. The transform has been corrected with the phase shift of the first shell. The solid line (- - ) is a bulk sample, the open circles (O०O०O) is a mesoporous sample with ordered walls, the crosses $(x \times x \times x)$ is a mesoporous sample with disordered walls. 


\subsubsection{Zirconia}

${ }^{18} \mathrm{O}$ tracer diffusion studies have been made on nanocrystalline samples prepared by sputtering of the metals and subsequent oxidation followed by compaction [81,82]. The particle sizes were $80-100 \mathrm{~nm}$ and the pure $\mathrm{ZrO}_{2}$ was in the monoclinic phase. The experiments on pure $\mathrm{ZrO}_{2}$ showed an interface diffusion coefficient some 3-4 orders of magnitude greater than in the crystallites, the latter having slightly higher activation energy. The diffusion coefficients are lower than for cubic stabilised zirconia (CSZ) and yttrium stabilised cubic zirconia (YSZ) crystals, however it must be remembered that the latter materials are heavily doped resulting in a high concentration of mobile oxide ion vacancies. The ${ }^{18} \mathrm{O}$ diffusion in nanocrystalline $\mathrm{ZrO}_{2}$ doped with $6.9 \% \mathrm{Y}_{2} \mathrm{O}_{3}$ also showed a grain boundary diffusion that was more than three orders of magnitude greater than that within the grains. Thus there is clear evidence that the grain boundaries in nanoionics offer fast paths for diffusion.

Several groups have measured the conductivity in nanocrystalline zirconia, however the most recent study by Kosacki and co-workers [83] took great care to produce well-characterised samples. These workers prepared highly textured YSZ thin films deposited on (001) $\mathrm{MgO}$ single crystals by pulsed laser ablation of a dense polycrystalline cubic $\mathrm{ZrO}_{2} / 9.5 \mathrm{~mol} \% \mathrm{Y}_{2} \mathrm{O}_{3}$ target. After deposition, the specimens were annealed ex-situ for $1 \mathrm{~h}$ at $700{ }^{\circ} \mathrm{C}$ in oxygen to establish stoichiometry. Both DC and $\mathrm{AC}$ impedance techniques measured the electrical conductivity parallel to the substrate surface. The results are shown in Fig. 6. The conductivity clearly increases with decreasing particle size. It was concluded the films were free of blocking grain boundaries and conduction parallel to the substrate was characterised by a single film/substrate interface. An enhancement in the in-plane conductivity was observed for films with thickness below $60 \mathrm{~nm}$ and is attributed to the transition from lattice to interface controlled diffusivity. It was estimated that the thickness of the interface layer was about $1.6 \mathrm{~nm}$ and

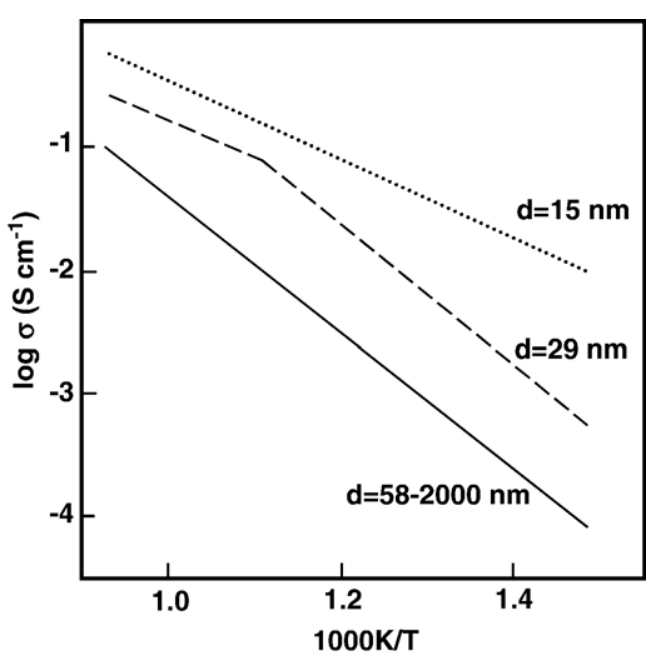

Fig. 6. The temperature dependence of the electrical conductivity for epitaxial YSZ thin films with different film thicknesses, deposited on $\mathrm{MgO}$ substrates (after [83]).

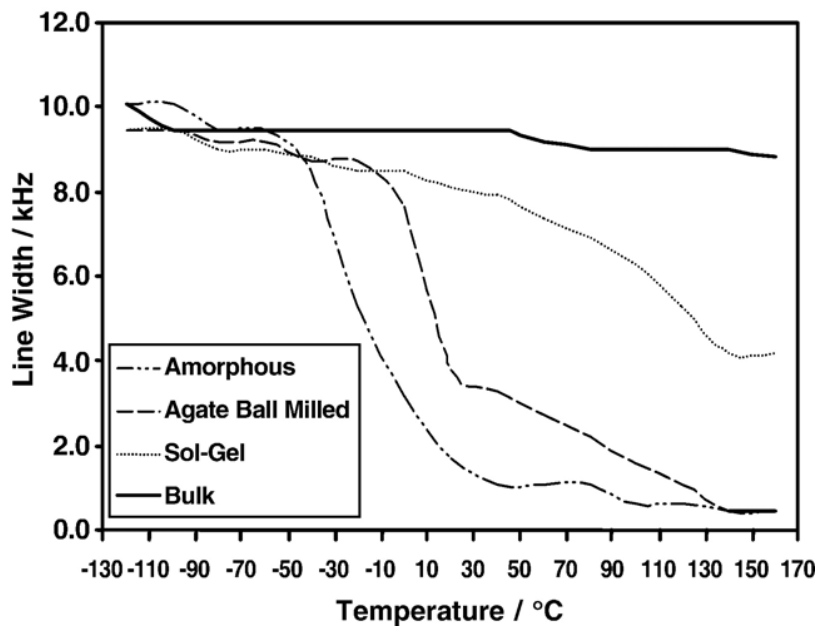

Fig. 7. The ${ }^{7} \mathrm{Li}$ NMR line width as a function of temperature for bulk, amorphous, sol-gel and ball milled $\mathrm{LiNbO}_{3}$.

interfacial conductivity was over three orders of magnitude larger than lattice limited conductivity, in agreement with the ${ }^{18} \mathrm{O}$ tracer measurements.

\subsubsection{Lithium niobate}

Although lithium niobate is a relatively complex system compared to the binary oxides and is not an intrinsic ionic conductor it has become a model for nanoionics due to the initial observation by Bork and Heitjans [84] of enhanced lithium ion conduction in ball-milled samples. This has led to several investigations of this system from both the structural aspects, discussed in Section 3.1.2, and ionic transport using both $\mathrm{Li}$ NMR and complex impedance spectroscopy [76-78,84]. The latest paper on this system reports new data and shows that there is now a consistent interpretation of the data [78].

The nature of lithium ion transport in nanocrystalline lithium niobate is encapsulated by Fig. 7 , the ${ }^{7} \mathrm{Li}$ NMR line width as a function of temperature $[57,77]$. There is virtually no narrowing of the NMR line for the bulk material over the temperature range investigated, showing the absence of $\mathrm{Li}^{+}$ion motion. In contrast, the amorphous sample and the ball-milled sample (50 nm particle size) show a dramatic narrowing due to fast $\mathrm{Li}^{+}$ ion motion. However, for the sol-gel prepared sample $(21 \mathrm{~nm}$ particle size) the extent of line narrowing is much less pronounced. The similarity between the behaviour of ballmilled and amorphous samples and the indications of significant fractions of amorphous regions from the EXAFS data led to the conclusion that the ionic transport in these samples was predominantly through the amorphous phase. This has been supported by more recent work, particularly the direct TEM observation of amorphous regions in ball-milled samples [78]. The origin of the enhanced $\mathrm{Li}^{+}$ion motion in the sol-gel sample is still not fully understood. It is clearly due to conduction along the interfaces, which the TEM shows are well ordered. Therefore it could be due to space charge effects at the surface of the grains, and an example of the model proposed by Maier and co-workers [85-87]. 


\section{Conclusions}

Some important conclusions can be drawn from the EXAFS work presented in this contribution. Firstly, there is the general point that the method of preparation strongly influences the nature of the microstructure of nanoionic materials. We have made this point in earlier papers based on EXAFS data $[36,50,56,58,73]$. Here we have reported new EXAFS data for zirconia taken at $17 \mathrm{~K}$, which confirms the low levels of disorder in sol-gel prepared samples and the very high levels of disorder in ball-milled samples. This disorder is due to the formation of large quantities of amorphous material during the ball-milling procedure, which the recent TEM study of lithium niobate [78] shows to be a characteristic of this preparative method. Secondly, we have shown from the work on mesoporous $\alpha-\mathrm{Fe}_{2} \mathrm{O}_{3}$ that EXAFS measurements can be used to probe the microstructure of nanoionics with complex architectures. There is now considerable activity in preparing nanocrystalline oxides with unusual shapes, such as tube [88] and hollow spheres [89], and EXAFS should prove to be a vital tool in their characterisation.

The recent transport studies of zirconia appear to be showing some consistency, with both conductivity and tracer data showing the enhanced oxide ion migration in the nanocrystals being due to transport along the grain boundaries. There is now a uniform picture for the transport in nanocrystalline lithium niobate where the greatly enhanced lithium ion transport in ballmilled samples is due to diffusion through the amorphous regions. More work is required to determine the precise origin of the more modest enhancement in sol-gel samples. The reasonable level of agreed interpretation of the transport data for these two model systems has evolved due to the use of more direct diffusion methods, ${ }^{18} \mathrm{O}$ tracer for zirconia and ${ }^{7} \mathrm{Li} \mathrm{NMR}$ for lithium niobate, in addition to conductivity measurements. The wider use of a combination transport measurement would help to resolve some of the problems and diversity of data in other nanoionics.

\section{Acknowledgements}

The author wishes to thank the EPSRC for financial support for work in this article through grant GR/S61881/01. We also wish to thank our colleagues at the University of Warwick, Professor Mark Smith and Mr. Luke O'Dell, for NMR data and staff of the Daresbury SRS, particularly Drs J.W.F. Mosselmans, S. Hayama and I. Harvey, for help and advice with the XAS measurements.

\section{References}

[1] M.C. Roco, JOM-J. Minerals Metals and Materials Soc., vol. 54, 2002, p. 22.

[2] H. Gleiter, Prog. Mater. Sci. 33 (1989) 223.

[3] H. Gleiter, Adv. Mater. 4 (1992) 474.

[4] A. Henglein, Chem. Rev. 89 (1989) 1061.

[5] H. Weller, Angew. Chem., Int. Ed. Engl. 32 (1993) 41.

[6] R.W. Siegel, G.E. Fougere, Nanostruct. Mater. 6 (1995) 205.

[7] H. Gleiter, Acta Mater. 48 (2000) 1.
[8] P. Moriarty, Rep. Prog. Phys. 64 (2001) 297.

[9] R. Würschum, U. Brossmann, H.-E. Schaefer, in: C.C. Koch (Ed.), Nanostructured Materials-Processing, Properties, and Applications, Noyes Publications, Norwich, UK, 2002, p. 267.

[10] A.S. Edelstein, R.C. Cammarata (Eds.), Nanomaterials: Synthesis, Properties and Applications, Institute of Physics, Bristol, UK, 2002.

[11] J.V. Stark, D.G. Park, I. Lagadic, K.J. Klabunde, Chem. Mater. 8 (1996) 1904.

[12] J.V. Stark, K.J. Klabunde, Chem. Mater. 8 (1996) 1913.

[13] Y. Sun, Y. Xia, Science 298 (2002) 2176.

[14] O.B. Koper, K.J. Klabunde, Chem. Mater. 9 (1997) 2481.

[15] R.W. Siegel, Annu. Rev. Mater. Sci. 21 (1991) 559.

[16] R. Würschum, Rev. Metall. 96 (2000) 1547.

[17] S.C. Tjong, H. Chen, Mater. Sci. Eng., R Rep. 45 (2004) 1.

[18] S. Chandra, Superionic Solids, Principles and Applications, NorthHolland, Amsterdam, 1981.

[19] M. Wakihara, O. Yamamoto (Eds.), Lithium Ion Batteries, Wiley-VCH, Weinheim, 1998.

[20] M.S. Whittingham, Chem. Rev. 104 (2004) 4271.

[21] J.-M. Tarascon, M. Armand, Nature 414 (2001) 359.

[22] C. Xu, J. Tamaki, N. Miura, N. Yamazoe, Sens. Actuators, B, Chem. 3 (1991) 147.

[23] S. Davis, A.V. Chadwick, J.D. Wright, J. Mater. Chem. 8 (1998) 2065.

[24] H.L. Tuller, Solid State Ionics 131 (2000) 143.

[25] N. Sata, K. Ebermann, K. Eberl, J. Maier, Nature 408 (2000) 946.

[26] P. Heitjans, S. Indris, J. Phys.: Condens. Matter. 15 (2003) R1257.

[27] A.V. Chadwick, Diffus. Fundam. 2 (2005) 44.

[28] H. Gleiter, in: N. Hansen, A. Horsewell, T. Lefferes, H. Lilholt (Eds.), Deformation of Polycrystals: Mechanisms and Microstructures, Riso National laboratory, Roskilde, 1981, p. 15.

[29] J. Ying, J. Aerosol Sci. 24 (1993) 315.

[30] D.H. Pearson, A.S. Edelstein, Nanostruct. Mater. 11 (1999) 1111.

[31] G. Gonzalez, J.A. Freites, C.E. Rojas, Scr. Mater. 44 (2001) 1883.

[32] P. Taneja, R. Chandra, R. Banerjee, P. Ayyub, Scr. Mater. 44 (2001) 1915.

[33] G.L. Messing, S.C. Zhang, G.V. Jayanthi, J. Am. Ceram. Soc. 76 (1993) 2707.

[34] C.J. Brinker, J.W. Scherer, Sol-Gel Science: The Physics and Chemistry of Sol-Gel Processing, Academic Press, Boston, USA, 1990.

[35] A.C. Pierre, Introduction to Sol-Gel Processing, Kluwer Academic Publishers, Boston, USA, 1998.

[36] A.V. Chadwick, G. Mountjoy, V.M. Nield, I.J.F. Poplett, M.E. Smith, J.H. Strange, M.G. Tucker, Chem. Mater. 13 (2001) 1219.

[37] S.R. Davis, A.V. Chadwick, J.D. Wright, J. Phys. Chem., B 101 (1997) 9901.

[38] H.J. Fecht, Nanostruct. Mater. 6 (1995) 33.

[39] C.C. Koch, Nanostruct. Mater. 9 (1997) 13.

[40] L.M. Cukrov, T. Tsuzuki, P.G. McCormick, Scr. Mater. 44 (2001) 1787.

[41] S. Indris, D. Bork, P. Heitjans, J. Mater. Synth. Process. 8 (2000) 245.

[42] J. Ding, T. Tsuzuki, P.G. McCormick, R. Street, J. Phys., D 29 (1996) 2365.

[43] E. Baburaj, K. Hubert, F. Froes, J. Alloys Compd. 257 (1997) 146.

[44] G. Scholz, R. Stosser, J. Klein, G. Silly, J.Y. Buzaré, Y. Laligant, B. Ziemer, J. Phys.: Condens. Matter. 14 (2002) 14, 2101.

[45] Y.-M. Chiang, E.B. Lavik, I. Kosacki, H.L. Tuller, J.Y. Ying, J. Electroceram. 1 (1997) 7.

[46] Th.E. Weirich, M. Winterer, S. Seifried, H. Hahn, F. Fuess, Th.E. Weirich, Ultramicroscopy 81 (2000) 263.

[47] R. Würschum, G. Soyez, H.-E. Schaefer, Nanostruct. Mater. 3 (1993) 225.

[48] T. Haubold, R. Birringer, B. Lengeler, H. Gleiter, Phys. Lett., A 135 (1989) 461.

[49] S. de Panfilis, F. d'Acapito, V. Haas, H. Konrad, J. Weissmüller, F. Boscherini, Phys. Lett., A 207 (1995) 397.

[50] A.V. Chadwick, G.E. Rush, in: P. Knauth, J. Schoonman (Eds.), Characterisation of Nanocrystalline Metal Oxides by XAS, in Nanocrystalline Materials, Kluwer, New York, USA, 2002, chapter 5.

[51] B.K. Teo, D.C. Joy (Eds.), EXAFS Spectroscopy; Techniques and Applications, Plenum Press, New York, USA, 1980.

[52] T.M. Hayes, J.B. Boyce, Solid State Phys. 37 (1982) 173.

[53] D.C. Koningsberger, R. Prins (Eds.), X-ray Absorption, Wiley, New York, USA, 1988. 
[54] Y.R. Wang, K.Q. Lu, D.H. Wang, Z.H. Wu, Z.Z. Fang, J. Phys.: Condens. Matter. 6 (1994) 633.

[55] Z. Qi, C. Shi, Y. Wei, Z. Wang, T. Liu, T. Hu, Z. Zhan, F. Li, J. Phys.: Condens. Matter. 13 (2001) 11503.

[56] G.E. Rush, A.V. Chadwick, I. Kosacki, H.U. Anderson, J. Phys. Chem., B 104 (2000) 9597.

[57] A.V. Chadwick, Solid State Ionics, (2006), in press.

[58] S.L.P. Savin, A.V. Chadwick, L.A. O’Dell, M.E. Smith, Solid State Ionics, (2006), in press

[59] P. Heitjans, Solid State Ionics 18/19 (1989) 50.

[60] J. Philibert, Atom Movement, Diffusion and Mass Transport in Solids, Les Éditions de Physique, Paris, France, 1991.

[61] H. Mehrer, in: P. Heitjans, J. Kärger (Eds.), Diffusion in Condensed MatterMethods, Materials, Models, Springer, Berlin, Germany, 2005, chapter 1.

[62] D.S. McPhail, J. Mater. Sci. 41 (2006) 873.

[63] P. Heitjans, A. Schirmer, S. Indris, in: P. Heitjans, J. Kärger (Eds.), Diffusion in Condensed Matter-Methods, Materials, Models, Springer, Berlin, Germany, 2005, chapter 9.

[64] A.V. Chadwick, J.C.S. Faraday I, J. Chem. Soc., Faraday Trans. 86 (1990) 1157.

[65] A.B. Lidiard, Handb. Phys. XX (1957) 246.

[66] F. Bénière, in: J. Hladik (Ed.), Physics of Electrolytes, Academic Press, London, UK, 1972, p. 203.

[67] A.V. Chadwick, Philos. Mag. A64 (1991) 983.

[68] J.R. McDonald (Ed.), Impedance Spectroscopy, Wiley, New York, USA, 1983.

[69] S.P.S. Badwal, S. Rajendran, Solid State Ionics 70/71 (1994) 83.

[70] J. Fleig, Solid State Ionics 131 (2000) 117.

[71] J.E. Bauerle, J. Phys. Chem. 30 (1969) 2657.

[72] H. Liu, L. Feng, X. Zhang, Q. Xue, J. Phys. Chem. 99 (1995) 332.
[73] A.V. Chadwick, M.J. Pooley, K.E. Rammutla, S.L.P. Savin, A. Rougier, J. Phys.: Condens. Matter. 15 (2003) 431.

[74] A.V. Chadwick, S.L.P. Savin, L.A. O'Dell, M.E. Smith, J. Phys.: Condens. Matter. 18 (2006) L163.

[75] T.D. Shen, C.C. Koch, T.L. McCormick, R.J. Nemanich, J.Y. Huang, J.G. Huang, J. Mater. Res. 10 (1995) 139.

[76] M. Wilkening, D. Bork, S. Indris, P. Heitjans, Phys. Chem. Chem. Phys. 4 (2002) 3246.

[77] A.V. Chadwick, M.J. Pooley, S.L.P. Savin, Phys. Status Solidi (c) 2 (2005) 302.

[78] P. Heitjans, M. Masoud, A. Feldhoff, M. Wilkening, Faraday Discuss. 134 (2006) 1.

[79] F. Jiao, A. Harrison, J.-C. Jumas, A.V. Chadwick, W. Kockelmann, P.G. Bruce, J. Am. Chem. Soc. 128 (2006) 5468.

[80] C. Suryanarayana, C.C. Koch, Hyperfine Interact. 130 (2000) 5.

[81] G. Knöner, K. Reimann, R. Röwer, H.-E. Schaefer, Proc. Natl. Acad. Sci. 100 (2003) 2870

[82] U. Brossman, G. Knöner, H.-E. Schaefer, R. Würschum, Rev. Adv. Mater Sci. 6 (2004) 7.

[83] I. Kosacki, T.C.M. Rouleau, P.F. Becher, J. Bentley, D.H. Lowndes, Solid State Ionics 176 (2005) 1319.

[84] D. Bork, P. Heitjans, J. Phys. Chem., B 102 (1998) 7303.

[85] J. Maier, Prog. Solid State Chem. 23 (1995) 171.

[86] J. Maier, Solid State Ionics 131 (2000) 13.

[87] W. Puin, S. Rodewald, R. Ramlau, P. Heitjans, J. Maier, Solid State Ionics $131(2000) 159$.

[88] D. Gong, C.A. Grimes, O.K. Varghese, W.C. Hu, R.S. Singh, Z. Chen, E.C Dickey, J. Mater. Res. 16 (2001) 3331.

[89] Y.D. Yin, R.M. Rioux, C.K. Erdonmez, S. Hughes, G.A. Somorjai, A.P. Alivisatos, Science 304 (2004) 711. 